\title{
Ricci-flat metrics and Killing-Yano tensors
}

\author{
Dmitri Bykov ${ }^{1,2, *}$ \\ ${ }^{1}$ Ludwig-Maximilians-Universität, Theresienstrasse 37, 80333 Munich, Germany \\ ${ }^{2}$ Steklov Mathematical Institute, Gubkina str. 8, 119991 Moscow, Russia
}

\begin{abstract}
We consider the problem of constructing Ricci-flat metrics on the total space of the canonical bundle over the del Pezzo surface of rank one. We analyze the so-called 'orthotoric metric' and its first-order deformation, whose existence is compatible with the Calabi-Yau theorem.
\end{abstract}

\section{Introduction}

We will be considering Calabi-Yau threefolds $\mathcal{M}$ :

- Complex manifolds of complex dimension three: $\operatorname{dim}_{\mathbb{C}} \mathcal{M}=3$

- Zero first Chern class: $c_{1}(\mathcal{M})=c_{1}(K)=0$

( $K$ is the canonical bundle $=$ bundle of 3-forms $\Omega \propto f(z) d z_{1} \wedge d z_{2} \wedge d z_{3}$ ), i.e. there exists a non-vanishing holomorphic 3-form $\Omega$

- Such manifolds are used for supersymmetric compactifications in supergravity $\left(\mathbb{R}^{3,1} \times \mathcal{M}\right)$, and serve as backgrounds for brane constructions $\left(A d S_{5} \times Y^{5}\right)$

It is easy to show that compact Calabi-Yau's do not admit Killing vectors (apart from trivial cases), therefore explicit metrics are difficult to construct. For this reason we will be considering non-compact Calabi-Yau's, which do have symmetries. In this case the geometry of such manifolds may often be studied explicitly. These non-compact Calabi-Yau's may be thought of as describing singularities of compact Calabi-Yau's.

We will now describe a particular class of non-compact Calabi-Yau manifolds that we will study. Let $X$ be a positively curved complex surface, $c_{1}(X)>0$. Here one should recall that $c_{1}(X)=\left[\frac{i}{2 \pi} R_{m \bar{n}} d z^{m} \wedge d \bar{z}^{\bar{n}}\right] \in H^{2}(X, \mathbb{R})$. We will be studying the case when

$$
\mathcal{M}=\text { Total space of the canonical bundle of } X=\text { "Cone over } X \text { ". }
$$

This is just like the prototypical $\mathbb{C}^{2} / \mathbb{Z}_{2}$-singularity (" $A_{1}$-singularity", given by the equation $x y=z^{2}$ ) may be resolved by gluing in a copy of $\mathbb{C P}^{1}$ at the origin. The metric on the resolved space is then the Eguchi-Hanson metric. (However, this corresponds to $\mathcal{M}$ of complex dimension 2.)

*e-mail: dmitri.bykov@physik.uni-muenchen.de,bykov@mi.ras.ru 


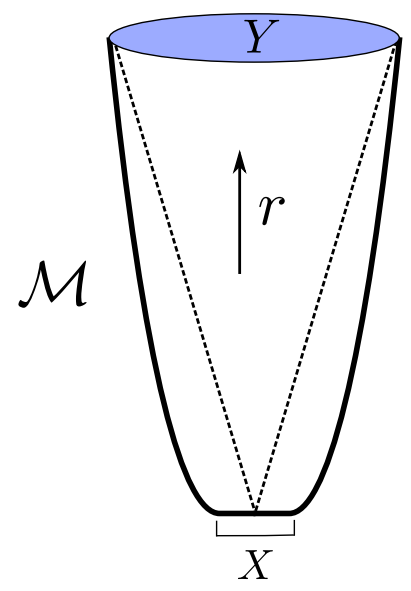

Figure 1: The corresponding singularity is pointlike and may be then resolved by gluing in a copy of $X$.

\subsection{First example. Calabi's ansatz.}

If $X$ admits a Kähler-Einstein metric, the metric on $\mathcal{M}$ may be found by means of an ansatz [1]

$$
\mathcal{K}=\mathcal{K}\left(|u|^{2} e^{K}\right),
$$

where $\mathcal{K}$ and $K$ are the Kähler potentials of $\mathcal{M}$ and $X$ respectively. The Ricci-flatness equation becomes in this case an ODE for the function $\mathcal{K}(x)$. For example, for $X=\mathrm{CP}^{2}$ one obtains in this way the (generalized) Eguchi-Hanson metric [2]. These metrics are asymptotically-conical, i.e. they have the form

$$
d s^{2}=d r^{2}+r^{2}\left(\widetilde{d s^{2}}\right)_{Y} \quad \text { at } \quad r \rightarrow \infty,
$$

where $\left(\widetilde{d s^{2}}\right)_{Y}$ is a Sasaki-Einstein metric on a 5D real manifold $Y$.

An important characteristic of a Kähler metric on $\mathcal{M}$ is the cohomology class $[\omega] \epsilon$ $H^{2}(\mathcal{M}, \mathbb{R})$ of the Kähler form. Since $\mathcal{M}$ is a total space of a line bundle, its cohomology is the same as that of the underlying surface $X$. Therefore, for instance for $X=\mathbb{C P}^{2}$ we have $H^{2}(\mathcal{M}, \mathbb{R}) \simeq \mathbb{R}$, but for $X=\mathbb{C P}^{1} \times \mathbb{C P}^{1}$ we have $H^{2}(\mathcal{M}, \mathbb{R}) \simeq \mathbb{R}^{2}$.

Calabi's ansatz gives a metric with a very particular and fixed $[\omega] \in H^{2}(\mathcal{M}, \mathbb{R})$. It turns out that in this case $[\omega] \in H_{c}^{2}(\mathcal{M}, \mathbb{R}) \subset H^{2}(\mathcal{M}, \mathbb{R})$, where $H_{c}^{2}$ is the compactly supported cohomology. By Poincaré duality, the group $H_{c}^{2}(\mathcal{M}, \mathbb{R}) \simeq H_{4}(\mathcal{M}, \mathbb{R}) \simeq H_{4}(X, \mathbb{R}) \simeq \mathbb{R}$ is one-dimensional.

\subsection{The Calabi-Yau theorem.}

The Calabi-Yau theorem [3], [4] states, however, that, at least for compact $\mathcal{M}$, there is a unique Ricci-flat metric in every Kähler class $[\omega] \in H^{2}(\mathcal{M}, \mathbb{R})$.

For the case of interest $\mathcal{M}$ is not compact, but rather asymptotically-conical, and in this case there exists a proposal for a CY theorem due to [5], [6]. Moreover, one has the decay estimates

$$
\begin{aligned}
& \left|g-g_{0}\right|_{g_{0}}=O\left(\frac{1}{r^{6}}\right) \quad \text { for } \quad[\omega] \in H_{c}^{2}(\mathcal{M}, \mathbb{R}) \\
& \left|g-g_{0}\right|_{g_{0}}=O\left(\frac{1}{r^{2}}\right) \quad \text { for } \quad[\omega] \in H^{2}(\mathcal{M}, \mathbb{R}) \backslash H_{c}^{2}(\mathcal{M}, \mathbb{R}),
\end{aligned}
$$


where $g_{0}$ is the conical metric. Such estimates were introduced for the case of ALE-manifolds in [7].

\subsection{Example. $X=\mathbb{C P}^{1} \times \mathbb{C P}^{1}$.}

The theory just described can be tested explicitly at the example of $X=\mathbb{C P}^{1} \times \mathbb{C P}^{1}$. The ansatz for the Kähler potential on the cone over $X$ is a generalized ansatz of Calabi constructed by [8], [9]:

$$
\mathcal{K}=a \log \left(1+\left|w^{2}\right|\right)+\mathcal{K}_{0}\left(\left|u^{2}\right|\left(1+\left|w^{2}\right|\right)\left(1+\left|x^{2}\right|\right)\right) .
$$

The resulting metric, indeed, has two parameters that define the cohomology class of the Kähler form $[\omega] \in H^{2}(\mathcal{M}, \mathbb{R})=\mathbb{R}^{2}$. These correspond to the sizes of the two spheres. The relevant Sasakian manifold $Y$ at $r \rightarrow \infty$ is the conifold $T^{11}=\frac{S U(2) \times S U(2)}{U(1)}$, and the decay at infinity agrees with the predicted one.

\section{The del Pezzo surface}

We will be interested in the next-to-simplest example when $X$ is the del Pezzo surface of rank one (also known as the Hirzebruch surface of rank one), i.e. the blow-up of $\mathbb{C P}^{2}$ at one point.

A blow-up means that we replace one point in $\mathbb{C P}^{2}$ by a sphere $\mathbb{C P}^{1}$. This $\mathbb{C P}^{1}$ 'remembers the direction', at which we approach the point. A 'good' metric on the new manifold should have two parameters, which describe the original size of the $\mathbb{C P}^{2}$ and the size of the glued in sphere $\mathbb{C P}^{1}$. The del Pezzo surface is a toric manifold, and the best way to think of it is via its moment polygon.
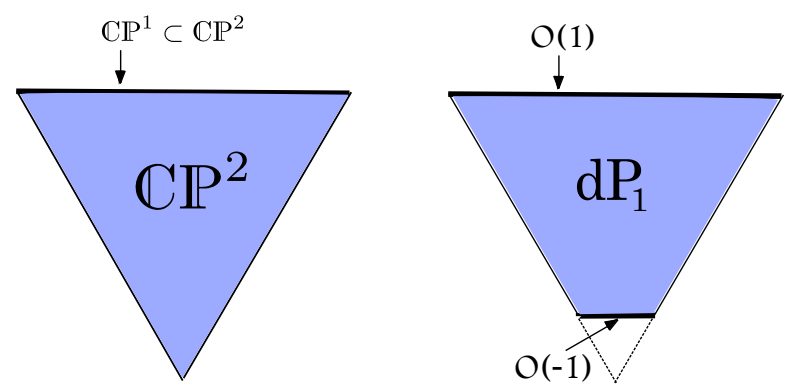

\subsection{Metrics on the cone and toric geometry}

A theorem of [10] says that there does not exist a Kähler-Einstein metric on $\mathbf{d P}_{1}$. How do we then construct a metric on the cone $\mathcal{M}$ over $\mathbf{d P}_{1}$ ? The only hope is to use its symmetries, which are those symmetries of $\mathbb{C P}^{2}$ that remain after the blow-up.

The relevant isometry group is $U(1) \times U(2)$, however for the moment let us focus on the toric $U(1)^{3}$ subgroup. Generally, the Kähler potential has the form

$$
\mathcal{K}=\mathcal{K}(\underbrace{\left|z_{1}\right|^{2}}_{=e^{t_{1}}}, \underbrace{\left|z_{2}\right|^{2}}_{=e^{t_{2}}}, \underbrace{\left|z_{3}\right|^{2}}_{=e^{t_{3}}}) .
$$

It is customary to introduce the symplectic potential $\mathcal{G}$ - the Legendre transform of the Kähler potential w.r.t. $t_{i}$ :

$$
\mathcal{G}\left(\mu_{1}, \mu_{2}, \mu_{3}\right)=\sum_{j=1}^{3} \mu_{i} t_{i}-\mathcal{K}
$$


Here $\mu_{i}=\frac{\partial \mathcal{K}}{\partial t_{i}}$ are the moment maps for the $U(1)^{3}$ symmetries of the problem. The metric on $\mathcal{M}$ has the form

$$
d s^{2}=\frac{1}{4} \mathcal{G}_{i j} d \mu^{i} d \mu^{j}+\left(\mathcal{G}^{-1}\right)^{i j} d \phi_{i} d \phi_{j}
$$

The Riemann tensor with all lower indices looks as follows:

$$
R_{\bar{m} j k \bar{n}}=-\sum_{s, t} \mathcal{G}_{n s}^{-1} \frac{\partial^{2} \mathcal{G}_{j k}^{-1}}{\partial \mu_{s} \partial \mu_{t}} \mathcal{G}_{t m}^{-1} .
$$

The domain, on which $\mathcal{G}$ is defined, is the moment polytope. The potential $\mathcal{G}$ has singularities at the boundaries of the polytope. For instance, for flat space $\mathbb{C}^{3}$ the polytope is the octant, and $\mathcal{G}$ has the form

$$
\mathcal{G}_{\text {flat }}=\sum_{k=1}^{3} \mu_{k}\left(\log \mu_{k}-1\right) .
$$

In general, at a boundary $\ell=0$ the potential behaves as $\mathcal{G}=\ell(\log \ell-1)+\ldots$

Quite generally, Kähler metrics on toric manifolds were constructed by [11]. They are built using Kähler quotients, and the corresponding symplectic potential exhibits the singularities just described.

In our problem we have more symmetry: $U(1) \times U(2)$ instead of $U(1)^{3}$. The Kähler potential is therefore

$$
\mathcal{K}=\mathcal{K}(\underbrace{|w|^{2}}_{=e^{t}}, \underbrace{\left|z_{1}\right|^{2}+\left|z_{2}\right|^{2}}_{=e^{s}}),
$$

which means that the metric is of cohomogeneity-2. This implies the following form of $\mathcal{G}$ :

$$
\begin{aligned}
& \mathcal{G}=\left(\frac{\mu}{2}+\tau\right) \log \left(\frac{\mu}{2}+\tau\right)+\left(\frac{\mu}{2}-\tau\right) \log \left(\frac{\mu}{2}-\tau\right)-\mu \log \mu+G(\mu, v) \\
& \mu=\mu_{1}+\mu_{2}, \quad \tau=\frac{\mu_{1}-\mu_{2}}{2}, \quad v=\mu_{3} .
\end{aligned}
$$

The Ricci-flatness equation is then a Monge-Ampère equation in two variables:

$$
e^{G_{\mu}+G_{v}}\left(G_{\mu \mu} G_{v \nu}-G_{\mu \nu}^{2}\right)=\mu
$$

The domain of definition is the moment polytope of the cone $\mathcal{M}$ :

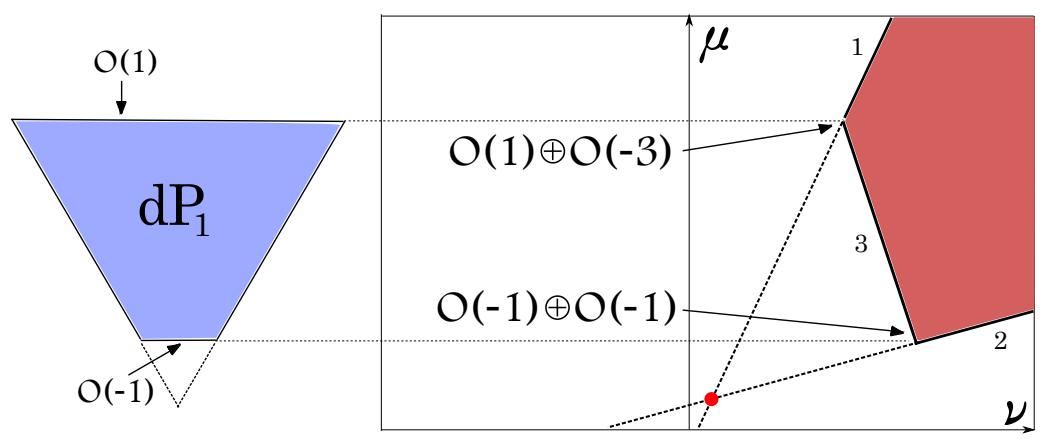




\subsection{The asymptotic behavior of $G$}

One can construct an exact solution of the above equation taking the conical ansatz for the metric $d s^{2}=d r^{2}+r^{2} \widetilde{d s^{2}}$. We make a change of variables $(\mu, v) \rightarrow\left(v, \xi=\frac{\mu}{v}\right)$ and look for $G$ in the form $\left(v \propto r^{2}\right)$

$$
G=3 v(\log v-1)+v P(\xi)
$$

One obtains an ODE for $P(\xi)$ that can be solved exactly. As a result,

$$
G=\sum_{i=0}^{2} \frac{\mu-\xi_{i} v}{1-\xi_{i}}\left(\log \left(\mu-\xi_{i} v\right)-1\right),
$$

where $\xi_{i}$ are the roots of the cubic polynomial $Q(\xi)=\xi^{3}-\frac{3}{2} \xi^{2}+d$. Varying $d$, one arrives at the Sasakian manifolds called $Y^{p, q}$ that were discovered in [12]. The topology of the underlying del Pezzo surface forces us to pick the manifold $Y^{2,1}$.

\subsection{Uniqueness}

The conical metric constructed above is singular at $r=0$. Constructing a smooth - resolved - metric is rather difficult. For the moment let us assume that, for a fixed moment polytope, we constructed one such metric with potential $G_{0}$. To check uniqueness, one can expand $G=G_{0}+H$ to first order in $H$ :

$$
\triangle_{G_{0}} H=0 \quad \Rightarrow \quad 0=\int d \mu d v H \triangle_{G_{0}} H \stackrel{?}{=}-\int d \mu d v(\nabla H)^{2}
$$

Whether we may integrate by parts depends on the behavior at infinity, where we have asymptotically

$$
\triangle_{G_{0}} H=0 \quad \rightarrow \quad-\frac{\partial}{\partial \xi}\left(Q(\xi) \frac{\partial H}{\partial \xi}\right)+\frac{\xi}{v} \frac{\partial}{\partial v}\left(v^{3} \frac{\partial H}{\partial v}\right)=0
$$

Substituting $H=v^{m} h(\xi)$, we get a Heun equation

$$
-\frac{d}{d \xi}\left(Q(\xi) \frac{d h}{d \xi}\right)+m(m+2) \xi h(\xi)=0
$$

Therefore one needs to estimate the spectrum of the Laplacian on $Y^{2,1}$. We have the following result:

Proposition. [13]

For the smallest non-zero eigenvalue $\lambda$ of the Laplacian $\Delta_{\xi}=-\frac{d}{d \xi}\left(Q(\xi) \frac{d h}{d \xi}\right)$, entering the equation $\triangle_{\xi} f+\lambda \xi f=0$, one has the lower bound $\lambda \geq 3$.

As a result, we obtain uniqueness of the metric for a given moment polytope. Therefore all potential moduli of the metric have to be related to the moduli of the polytope, which in turn are the Kähler moduli.

\section{Killing-Yano forms.}

One approach to the explicit construction of a metric is to require that it admit a conformal Killing-Yano form (CKYF). First we recall some basic examples of conditions that lead to the reduction of the holonomy group:

$$
\begin{array}{llll}
\nabla_{i} \xi_{j}=0 & \Rightarrow & \multicolumn{2}{c}{\text { Reduced holonomy }} \\
\nabla_{i} \xi_{j}-\nabla_{j} \xi_{i}=0 & \Rightarrow & \xi=d \chi \\
\nabla_{i} \xi_{j}+\nabla_{j} \xi_{i}=0 & \Rightarrow & \text { Killing vector }
\end{array}
$$


The Killing-Yano form $\omega_{i j} d x^{i} \wedge d x^{j}$ is defined by the equation

$$
\nabla_{i} \omega_{j k}+\nabla_{j} \omega_{i k}=0
$$

The conformal Killing-Yano form is defined analogously:

$$
\nabla_{i} \omega_{j k}+\nabla_{j} \omega_{i k}-\text { trace parts }=0
$$

For the geodesic equation Killing tensors imply the existence of conserved quantities, polynomial in momenta (hence not directly related to isometries). These additional conserved quantities lead, for instance, to the integrability of the geodesic equation in the Kerr metric [14-17]. Note also that the variables in the equation for linearized perturbations around the Kerr metric separate for the same reason, and lead to the Heun equation as well. Many of the known (higher-dimensional) black hole solutions (Myers-Perry, etc.) admit Killing-Yano tensors (see the reviews [18-20].

Killing-Yano tensors have also been investigated in the context of Kähler geometry [21]. On a Kähler manifold we may expand $\omega=\omega^{(2,0)} \oplus \omega^{(1,1)} \oplus \omega^{(0,2)}$. The situation when $\omega$ is Hermitian, i.e. $\omega^{(2,0)}=0$, is especially simple. Introducing the 'shifted' form $\Omega_{a \bar{b}}=\omega_{a \bar{b}}-h g_{a \bar{b}}$ $\left(h=g^{a \bar{b}} \omega_{a \bar{b}}\right)$, one gets the equation [22]

$$
\nabla_{a} \Omega_{b \bar{c}}=-2 g_{a \bar{c}} \partial_{b} h
$$

The tensor $\Omega$ has various names, such as Hamiltonian two-form, twistor form, etc. One can show that its eigenvalue functions $x_{i}$ have orthogonal gradients. They can be related to the 'moment map' variables $\mu_{i}$ corresponding to holomorphic isometries via the interesting formula:

$$
\prod_{k=1}^{n}\left(\vartheta-x_{k}\right)=\sum_{k=0}^{n} \vartheta^{k} \mu_{k+1}
$$

\subsection{The orthotoric metric.}

At the end of the day the metric admitting a tensor $\Omega$ has the form (we set $x_{1}=x, x_{2}=y$, then $\mu=x y, v=x+y$ )

$$
d s^{2}=x y g_{\mathbb{C P}^{1}}+(x-y)\left(\frac{d x^{2}}{P_{1}(x)}+\frac{d y^{2}}{P_{2}(y)}\right)+\text { angular part }
$$

We call this metric the 'orthotoric metric'. We see that the variables separated. The requirement of Ricci-flatness fixes the functions $P_{1}, P_{2}$ to be cubic polynomials (one of which we encountered before):

$$
P_{1}(x)=x^{3}-\frac{3}{2} x^{2}+c \quad P_{2}(y)=y^{3}-\frac{3}{2} y^{2}+d .
$$

The domain is $x \leq x_{\min }, y \in\left[y_{1}, y_{2}\right]$. If we further require that the topology is that of the cone over $\mathbf{d} \mathbf{P}_{1}$, the constants $c$ and $d$ are uniquely fixed. This metric was also obtained by [23], [24] and was extensively studied in [25].

The point is that the requirements of

(a) Ricci-flatness

(b) Cone over $\mathbf{d} \mathbf{P}_{1}$ topology

(c) CKYF of type $(1,1)$

completely fix the metric.

According to the Calabi-Yau theorem, however, the metric should contain additional parameters, corresponding to the deformation of the moment polytope. Altogether there are 2 parameters, since $H^{2}(\mathcal{M}, \mathbb{R})=\mathbb{R}^{2}$. 


\subsection{Deformation of the metric and the CKYF.}

One parameter is somewhat 'trivial', as it corresponds to a rescaling of the metric. We can still look for the other non-trivial parameter, which corresponds to the following deformation:

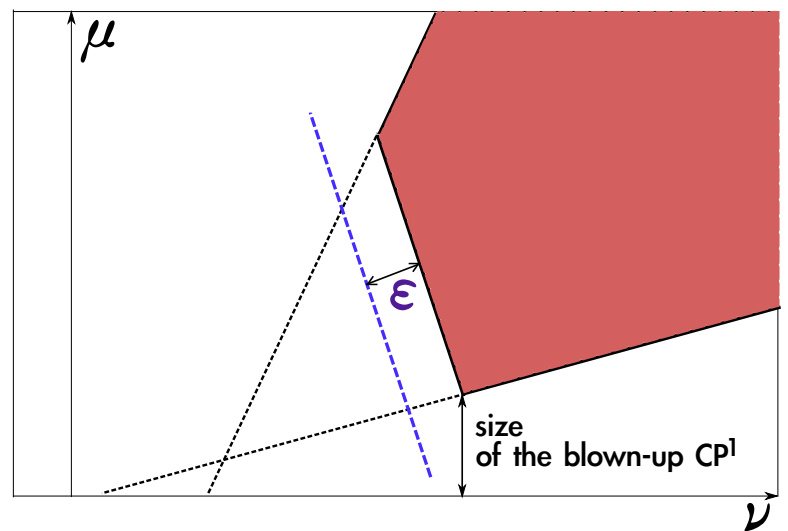

In the equation $\triangle_{G_{0}} H=0$, if we substitute the orthotoric potential $G_{0}$, the variables separate:

$$
\frac{1}{x} \frac{\partial}{\partial x}\left(P_{1}(x) \frac{\partial H}{\partial x}\right)-\frac{1}{y} \frac{\partial}{\partial y}\left(P_{2}(y) \frac{\partial H}{\partial y}\right)=0
$$

The unique solution compatible with the deformation of the moment polytope is

$$
H(x, y)=\epsilon \int_{x}^{\infty} \frac{d \hat{x}}{P_{1}(\hat{x})} .
$$

For large $x$ one has $H(x, y)=\frac{\epsilon}{2 x^{2}}+\ldots$, and for the metric this implies the asymptotic behavior $\left|g-g_{0}\right|_{g_{0}}=O\left(\frac{1}{r^{6}}\right)$. This implies that the variation of the Kähler form has the property $[\delta \omega] \in H_{c}^{2}(\mathcal{M}, \mathbb{R})$.

The next question is: what happens to the Killing-Yano form? If it is deformed, it must acquire a non-zero $(2,0)$ part, i.e. $\omega^{2,0}=\omega_{m n} d z^{m} \wedge d z^{n} \neq 0$. On a Calabi-Yau manifold one has a nowhere vanishing three-form $\Omega_{\text {mnp }} d z^{m} \wedge d z^{n} \wedge d z^{p}$, and one can construct the 'inverse' 3-vector $\widetilde{\Omega}^{m n p} \partial_{m} \wedge \partial_{n} \wedge \partial_{p}$. We can then dualize $\omega^{2,0}$ to obtain a vector field $\omega^{p}:=\widetilde{\Omega}^{m n p} \omega_{m n}$.

Using the fact that $\mathcal{M}$ is Ricci-flat, we can show that $\omega^{p}$ has to satisfy a rather stringent requirement

$$
R_{m p \bar{k}}^{n} \omega^{p}=0
$$

As we mentioned earlier, on a toric manifold the curvature tensor is $R_{\bar{m} j k \bar{n}}=$ $-\sum_{s, t} \mathcal{G}_{n s}^{-1} \frac{\partial^{2} \mathcal{G}_{j k}^{-1}}{\partial \mu_{s} \partial \mu_{t}} \mathcal{G}_{t m}^{-1}$. Using the explicit expression for the orthotoric potential $\mathcal{G}$, we can show that the only solution is $\omega^{p}=0$. The final result may be summarized as follows:

Proposition. [13]

There exists a first-order deformation of the orthotoric metric that preserves Ricciflatness and corresponds to a deformation of the moment polytope. Moreover, the deformation of the Kähler form has the property $[\delta \omega] \in H_{c}^{2}(\mathcal{M}, \mathbb{R})$. The deformed metric does not possess a conformal Killing-Yano tensor. 


\section{Summary.}

- Metrics on non-compact Calabi-Yau manifold can be sometimes constructed explicitly

- Examples in $\operatorname{dim}_{\mathbb{C}} \mathcal{M}=3$ : Cones over $\mathbb{C P}^{2}, \mathbb{C P}^{1} \times \mathbb{C P}^{1}$

- More complicated cases with conformal Killing-Yano tensors

- In the case of the cone over $\mathbf{d P}_{1}$ the corresponding metric is not the most general one, predicted by the Calabi-Yau theorem

- One can explicitly construct a first-order deformation of the metric

- Some of the questions, which are yet to be answered, are:

What is the significance of the explicitly known (orthotoric) metric? Can one obtain a closed expression for the metric in the general case, or in other special cases?

Acknowledgements. I would like to cordially thank the organizers of "Quarks 2018" for the invitation. I am indebted to A.A.Slavnov and to my parents for support and encouragement.

\section{References}

[1] E. Calabi, Annales Scientiques de 1 École Normale Supérieure 12, 268-294 (1979)

[2] T. Eguchi, A. J. Hanson, Phys.Lett. B 74, 249 (1978)

[3] E. Calabi, in: Algebraic Geometry and Topology. A Symposium in Honor of S. Lefschetz (Princeton University Press, 1957), 78-89.

[4] S.-T. Yau, Comm. Pure and App. Math. 31, 339-411 (1979)

[5] C. van Coevering, Journal Math. Ann. 347:3, 581-611 (2010)

[6] R. Goto, J. Math. Soc. Japan 64:3, 1005-1052 (2012)

[7] D. Joyce, Ann. Global Anal. Geom. 19:1, 55-73 (2001)

[8] P. Candelas, X. C. de la Ossa, Nucl. Phys. B 342, 246 (1990)

[9] L. A. Pando Zayas, A. A. Tseytlin, Phys. Rev. D 63, 086006 (2001)

[10] G. Tian, S.-T. Yau, Comm. Math. Phys. 112:1, 175-203 (1987)

[11] V. Guillemin, J. Differential Geom. 40:2, 285-309 (1994)

[12] J. P. Gauntlett, D. Martelli, J. Sparks, D. Waldram, Adv. Theor. Math. Phys. 8:4, 711 (2004)

[13] D. Bykov, arXiv:1712.07227 (2017).

[14] B. Carter, Comm. Math. Phys. 10, 280-310 (1968)

[15] M. Walker, R. Penrose, Comm. Math. Phys. 18, 265-274 (1970)

[16] R. Floyd, (Ph.D. thesis, Birkbeck College, University of London, 1973)

[17] S. A. Teukolsky, Class. Quant. Grav. 32:12, 124006 (2015)

[18] O. P. Santillan, J. Math. Phys. 53, 043509 (2012)

[19] Y. Chervonyi and O. Lunin, JHEP 1509, 182 (2015)

[20] V. Frolov, P. Krtous and D. Kubiznak, Living Rev. Rel. 20:1, 6 (2017)

[21] A. Moroianu, U. Semmelmann, Ann. Sc. Norm. Super. Pisa Cl. Sci. (5) 2:4, 823-845 (2003)

[22] V. Apostolov, D. M. J. Calderbank, P. Gauduchon, J. Differential Geom. 73:3, 359-412 (2006)

[23] T. Oota, Y. Yasui, Phys. Lett. B 639, 54 (2006)

[24] W. Chen, H. Lu, C. N. Pope, Nucl. Phys. B 762, 38 (2007)

[25] D. Martelli, J. Sparks, J. Geom. Phys. 59, 1175 (2009) 